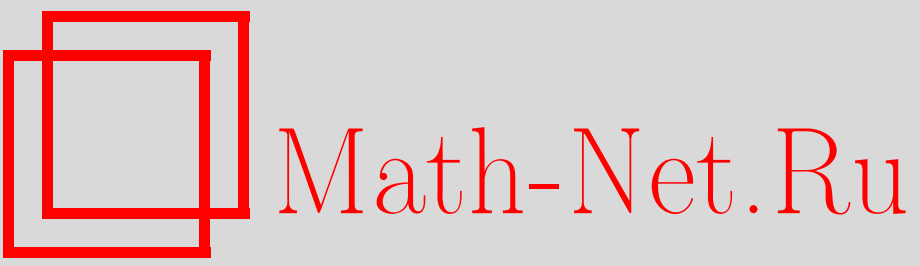

Г. И. Баренблатт, Турбулентные пограничные слои при очень больших числах Рейнольдса, УМН, 2004, том 59, выпуск 1, 45-62

DOI: https://doi.org/10.4213/rm700

Использование Общероссийского математического портала Math-Net.Ru подразумевает, что вы прочитали и согласны с пользовательским соглашением

http://www.mathnet.ru/rus/agreement

Параметры загрузки:

IP: 18.234 .156 .22

26 апреля 2023 г., 15:36:54 


\title{
ТУРБУЛЕНТНЫЕ ПОГРАНИЧНЫЕ СЛОИ ПРИ ОЧЕНЬ БОЛЬШИХ ЧИСЛАХ РЕЙНОЛЬДСА
}

Г. И. БАРЕНБЛАТТ

\begin{abstract}
Андрей Николаевич Колмогоров твердо стоял на позиции, что в отсутствие строгой, замкнутой в себе теории турбулентных жидкостей и газов следует опираться на гипотезы, получаемые из обработки экспериментальных данных. В начале работы обсуждается гипотеза полной автомодельности, лежащая в основе вывода широко известного универсального (не зависящего от числа Рейнольдса) логарифмического закона Кармана-Прандтля для распределения скорости в турбулентном сдвиговом потоке. Показано, что эта гипотеза не подтверждается экспериментами. Взамен выдвигается гипотеза неполной автомодельности, которая приводит к степенному закону, зависящему от числа Рейнольдса. Показано, что этот закон хорошо согласуется с экспериментами для важнейших классов сдвиговых турбулентных течений (течения в трубах и пограничных слоях).

Библиография: 27 названий.
\end{abstract}

1. Турбулентность - состояние вихревого движсения жидкости, в котором свойства полей потока (скорости, давления и т. д.) меняются в пространстве и времени случайным образом.

Турбулентные потоки окружают нас - в атмосфере, в океанах, в инженерных и естественных системах. Впервые распознанная, изученная и даже окрешенная Леонардо да Винчи, турбулентность в течение более чем столетия изучалась учеными и инженерами, в том числе гигантами - Андреем Николаевичем Колмогоровым, В. Гейзенбергом, Г. И. Тейлором, Л. Прандтлем, Т. фон Карманом. Любой успех в широком наборе тем, от хаоса и фракталов до теории поля, и любой рост в скорости и параллелизации компьютеров объявлялся вводяшим в решение "проблемы турбулентности”. Однако турбулентность - явление, знакомое теперь каждому, - остается величайшей проблемой в прикладной математике и физике. Турбулентность при очень больших числах Рейнольдса (часто назьваемая развитой турбулентностью) была, согласно широко распространенному мнению, одной из более счастливых провинший королевства турбулентности, поскольку многие думали, что два основных результата в этой области надежно установлены и войдут в основном в нетронутом виде в будущую чистую самодостаточную теорию турбулентности. Эти результаты - универсальньй логарифмический закон фон Кармана-Прандтля для ограниченных стенками турбулентных потоков со сдвигом (иногда называемых сдвиговьм течением или потоком с градиентом скорости) [1], [2] и масштабные законы Колмогорова-Обухова 
для локальной структуры развитых турбулентных потоков, знаменитый закон К-41 $[3],[4]$.

Начало фундаментальных исследований турбулентных потоков при очень больших числах Рейнольдса может быть датировано очень точно: это была лекция Т. фон Кармана "Механическое подобие и турбулентность" на третьем международном конгрессе по прикладной механике в Стокгольме в конце августа 1930 г. [1]. Фон Карман начал свою лекцию следуюшим обшим утверждением:

"Наше экспериментальное знание внутренней структуры турбулентных потоков недостаточно для создания надежных оснований рационального теоретического вычисления скорости распределения и лобового сопротивления в так называемом гидравлическом потоке состояний. Многочисленные полуэмпирические формулы, например, попытка ввести турбулентные коэффициенты лобового сопротивления, неспособны удовлетворить ни теоретика, ни практика . . . Вместо этого я ограничусь уяснением того, что может быть достигнуто на основании чистой гидродинамики, если определенные гипотезы вводятся в связи с определенными основными вопросами".

Более чем пятьюдесятью годами позже, обозревая в конце жизни начало своей работы по турбулентности, Андрей Николаевич сказал [5; с. 421]:

“... Мне стало ясно, что трудно надеяться на создание замкнутой в себе чистой теории [турбулентных потоков жидкостей и газов] . . За отсутствием такой теории придется опираться на гипотезы, получаемые из обработки экспериментальных данных ... Непосредственной экспериментальной работой я не занимался, но потратил много энергии на расчетную и графическую обработку данных других исследователей”.

Таким образом, и фон Карман, и Андрей Николаевич думали, что нереалистично ожидать создания чистой замкнутой в себе общей теории турбулентности за реальное время. Оба они считали реалистичным и разумным путем надеяться на гипотезы, подсказываемые экспериментами, и поэтому справедливые только для специальных классов потоков.

Что мы можем сказать теперь, через много десятков лет? Очень мало что изменилось, если вообще что-нибудь, в отношении чистой общей теории. Удручает, что, несмотря на тяжелую работу армии ученых в течение более чем века, про турбулентность почти ничего не стало известно на основе первопринципов, а именно, уравнений Навье-Стокса и уравнения неразрьвности.

Однако кое-что изменилось, и изменилось решительно. Сейчас больший объем гораздо более надежных экспериментальных данных стал общедоступньм. Обрабатывая эти данные, мы можем уточнять (и изменять при необходимости) базовые гипотезы и основанные на них специальные теории.

В настояшее время трудно обсуждать локальную структуру турбулентности при очень высоких числах Рейнольдса. Причина проста: чистая лабораторная экспериментальная база данных еше недостаточна для того, чтобы делать определенные выводы. Геофизические (атмосферные и океанические) данные недостаточно чисты. Лично я верю, что K-41, масштабные законы Колмогорова-Обухова в их классической формулировке, описьвают широкий класс жидкостных потоков в природе. 
Андрей Николаевич Колмогоров, А. М. Обухов, и, несколько позже, В. Гейзенберг и Л. Онзагер - какие великие имена! - выбрали эту тему, весьма необычную для того времени, и установили эти законы. Такая красота не может быть бесполезной!

В этой лекции я буду обсуждать ограниченные стенками турбулентные потоки со сдвигом (фон Карман называл их гидравлическими потоками состояний) при очень больших числах Рейнольдса. Работа, о которой я говорю, была выполнена в основном в 1991-2002 гг. А. Дж. Чориньм, В. М. Простокишиным и мною. Читатель может обратиться к статьям [6]-[9], которые содержат и другие, более ранние ссылки.

Мы утверждаем, что общедоступные экспериментальные данные, полученные в последние десятилетия, противоречат основным гипотезам, предложенным Т. фон Карманом. Следовательно, универсальный логарифмический закон не вполне верен, и от него нужно отказаться. Последующие теории, основанные на универсальном логарифмическом законе, должны быть пересмотрены. Вместо этого закона мы предлагаем масштабньй закон, зависящий от числа Рейнольдса, для распределения скорости в потоке со сдвигом, ограниченном стенками, и соответствующий закон лобового сопротивления.

2. Мы переходим теперь к проблеме, представляющей для нас непосредственный интерес, а именно, к статистически устойчивым ограниченным стенками турбулентным потокам со сдвигом. Среди таких потоков много практически важных потоков, как, например, потоки в трубах и каналах и пограничные слои.

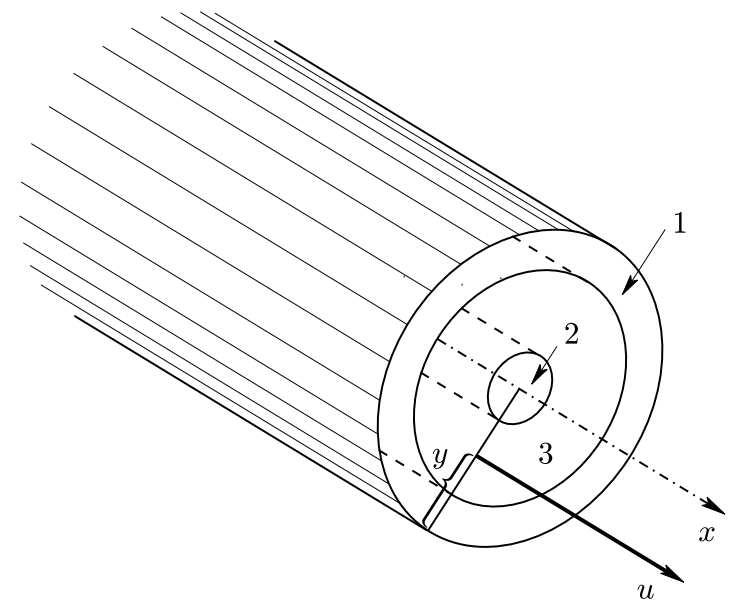

Рис. 1. Поток в длинной цилиндрической трубе; структура при большом числе Рейнолшдса: 1) вязкий подслой, 2) область вблизи оси, и 3) промежуточная область

Потоки в цилиндрических трубах (рис. 1) дают поучительный пример. У нас - та же ясная цель и полностью определенные задачи, что и сформулированные фон Карманом в его лекции: получить математическое выражение для скорости распределения в промежуточной области потока и для коэффициента лобового сопротивления. Слово "промежуточный" для потоков в трубах означает область между "вязким подслоем," примыкающим к стене, в котором градиенты скорости так высоки, что вязкое напряжение сравнимо с напряжением, создаваемьм турбулентными вихрями, и еше 
область непосредственно вблизи оси трубы. Для других потоков, ограниченных стенками, внешняя граница промежуточной области будет определена в надлежашем месте.

Гипотеза, предложенная фон Карманом, была выражена им в следуюшей прямой фором:

"На основе этих полностью экспериментально установленных фактов мы делаем предположение, что вне непосредственной окрестности стены распределение скорости среднего потока не зависит от вязкости".

Ясно, что градиент скорости $\partial_{y} u$, а не сама скорость $u$, предполагалась не зависящей от вязкости; по-видимому, это было явно сформулировано Ландау [10].

Рассмотрим теперь вывод распределения скорости в промежуточной области, основанньй на этой гипотезе. Градиент средней скорости $\partial_{y} u$ зависит от следующих аргументов: от трансверсальной координаты $y$ (расстояние от стены), от напряжения сдвига на стене $\tau$, от диаметра трубы $d$ и от свойств жидкости: ее кинематической вязкости $\nu$ и плотности $\rho$. Рассматривается именно градиент скорости $\partial_{y} u$, a не сама скорость $u$, потому что значения $u$ на любом расстоянии от стены зависят от потока вблизи стены (вязкий подслой), в котором используемые в дальнейшем предположения асимптотического характера не вьполняются. Таким образом,

$$
\partial_{y} u=f(y, \tau, d, \nu, \rho) \text {. }
$$

Следуя фон Карману и Прандтлю, мы вводим масштаб вязкой длины

$$
\delta=\nu / u_{*}, \quad \text { где } u_{*}=(\tau / \rho)^{1 / 2} .
$$

Величина $u_{*}$ называется динамической скоростью или скоростью “трения”. Размерностньй анализ дает

$$
\partial_{y} \phi=\frac{1}{y} \Phi\left(\frac{y}{\delta}, \frac{d}{\delta}\right), \quad \phi=\frac{u}{u_{*}} .
$$

Кроме того, размерностный анализ показывает, что $d / \delta$ - функция от традиционного числа Рейнольдса $\operatorname{Re}=\bar{u} d / \nu$, где $\bar{u}-$ средняя скорость, т.е. полный поток за единичное время, отнесенньй к площади поперечного сечения трубы. Таким образом, соотношение (3) может быть переписано в виде

$$
\partial_{y} \phi=\frac{1}{y} \Phi(\eta, \operatorname{Re}), \quad \eta=\frac{y}{\delta}=\frac{u_{*} y}{\nu} .
$$

Ламинарньй подслой занимает несколько десятков вязких длин, и поэтому для очень больших чисел Рейнольдса аргумент $y / \delta$ велик в промежуточной области. Основная гипотеза фон Кармана состоит в том, что вязкость не влияет на распределение скорости (на самом деле - градиента скорости) в промежуточной области. Между тем, вязкость входит в оба аргумента функции $\Phi$ в (4). Таким образом, в соответствии с гипотезой фон Кармана, масштаб вязкой длины $\delta$ должен исчезнуть из итогового соотношения, и функция $\Phi$ должна быть заменена постоянной, а именно, $\Phi=1 / \kappa$. Постоянная $\kappa$ была позже названа "постоянной Кармана". Подставляя $\Phi=1 / \kappa$ в (4), получаем

$$
\partial_{y} \phi=\frac{1}{\kappa y}, \quad \phi=\frac{u}{u_{*}} .
$$


Последующее интегрирование дает “универсальньй” (не зависяший от числа Рейнольдса) логарифмический закон фон Кармана-Прандтля для распределения скорости:

$$
\phi=\frac{u}{u_{*}}=\left[\frac{1}{\kappa} \ln \eta+C\right], \quad \eta=\frac{u_{*} y}{\nu} .
$$

Здесь постоянная $C,-$ и это тоже выглядит логически непротиворечивым, но на самом деле существенньм дополнительным предположением, - конечна и не зависит от числа Рейнольдса. По самой логике вьвода, постоянные $\kappa$ и $C$ должны совпадать во всех высококачественных экспериментах.

Однако в течение более чем шести десятилетий экспериментальная информация аккумулирует убедительные сомнения в универсальном логарифмическом законе (6). Мы подчеркиваем, что единственное место в вьводе соотношения (6), которое может вызвать сомнения, - это основная гипотеза фон Кармана. Действительно, даже если допустить чрезвычайно свободньй подход к постоянным $\kappa$ и $C$ (для $\kappa$ предлагались значения от 0.38 до 0.44 , т.е. для $1 / \kappa$ от 2.25 до 2.65 , а для $C$ - от 4.1 до 6.3 ), то область справедливости закона (6) оказьвается очень короткой. Здесь достаточно упомянуть данные, представленные в книге [11; гл. 3, раздел 5.3] , и в особенности еше более новые данные для потоков в трубах, полученные М. Загарола в Принстоне [12] (см. рис. 2). Хорошо видно расщепление кривых, соответствующих различным значениям числа Рейнольдса, и отклонение от прямой линии, представляюшей на плоскости $\ln \eta, \phi$ универсальный логарифмический закон, носит систематический характер.

3. Перед тем как двигаться дальше, сделаем следуюшее полезное общее замечание о масштабных законах. Рассмотрим физическое соотношение (т.е. справедливое для всех наблюдателей, имеюших другую величину единиц измерения)

$$
a=f\left(a_{1} \cdots a_{k}, b\right)
$$

где аргументы $a_{1}, \ldots, a_{k}$ имеют независимые размерности, причем размерности $a$ и $b$ могут быть выражены через размерности величин $a_{1}, \ldots, a_{k}$ :

$$
[a]=\left[a_{1}\right]^{p} \cdots\left[a_{k}\right]^{r}, \quad[b]=\left[a_{1}\right]^{p_{1}} \cdots\left[a_{k}\right]^{r_{1}} .
$$

Соотношение (7) можно представить в универсальной, безразмерной форме, которая вьполняется для всех наблюдателей, имеюших единицы измерения другой величины:

$$
\Pi=\Phi\left(\Pi_{1}\right), \quad \text { где } \Pi=\frac{a}{a_{1}^{p} \cdots a_{k}^{r}}, \quad \Pi_{1}=\frac{b}{a_{1}^{p_{1}} \cdots a_{k}^{r_{1}}} .
$$

Теперь, если значение параметра $\Pi_{1}$, для определенности, велико и если существует ненулевой конечный предел $\Phi\left(\Pi_{1}\right)$ при $\Pi_{1} \rightarrow \infty$, то закон $(7)$ при достаточно большом $\Pi_{1}$ может быть представлен как масштабньй закон:

$$
a=C a_{1}^{p} \cdots a_{k}^{r}
$$

где все показатели могут быть получены из размерностного анализа и величина $b$ не оказывает влияния на явление. Мы называем такие случаи полнымм подобием (ср. установившийся термин “подобие по числу Рейнольдса"). Что, однако, можно сказать, если такой конечньй ненулевой предел не существует? Вообще говоря, ничего. 


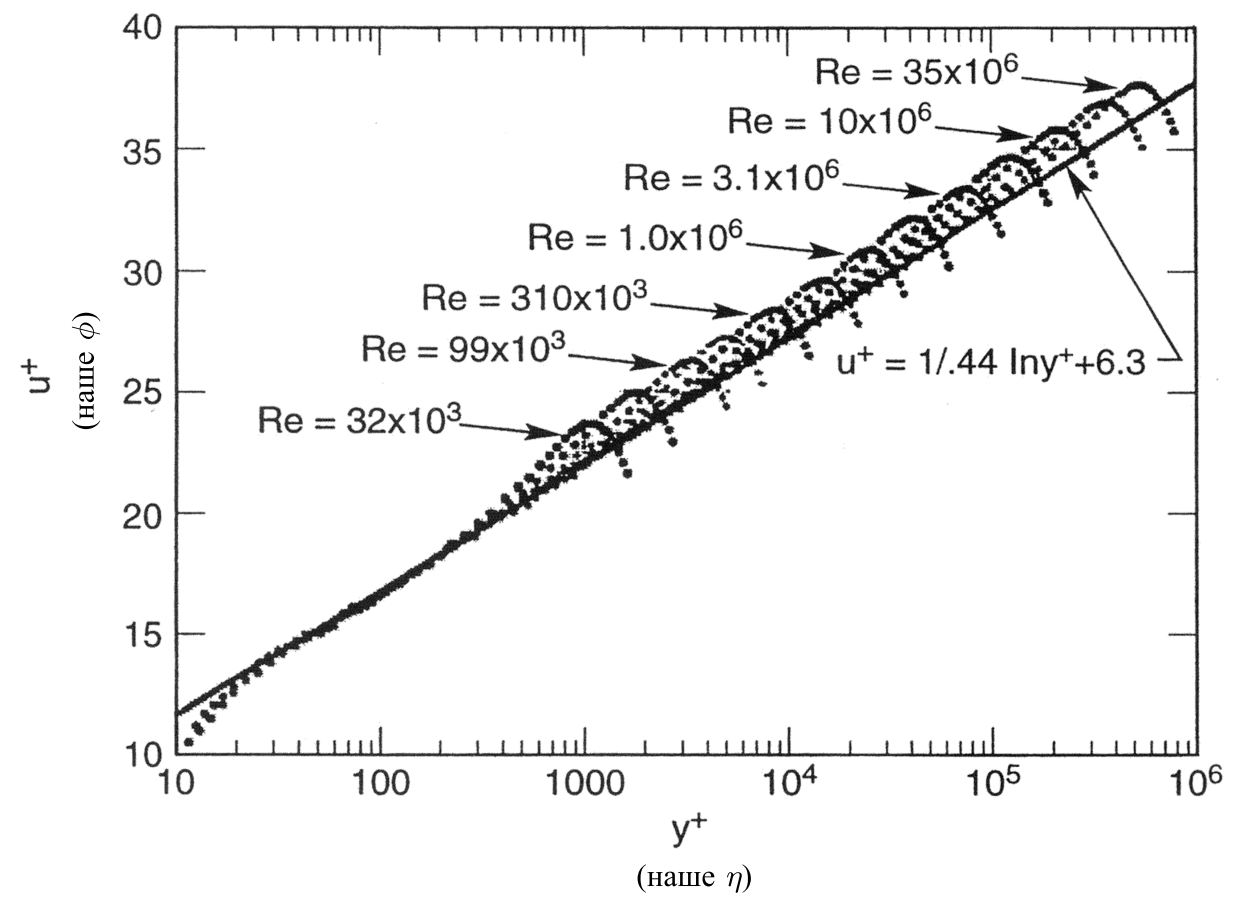

Рис. 2. Принстонские данные (Загарола [12]), полученные в трубе высокого давления, подтверждают расщепление экспериментальных данных в соответствии с их числами Рейнолшдса и подъем кривых над их огибающей в $(\ln \eta, \phi)$-плоскости. Сплошная линия - огибающая; кривые поворачивают в центре трубы. Расщепление и форма кривых согласованы с масштабным законом и не согласуются с универсальным логарифомическим законом фон Кармана-Прандтля

Однако есть важньй случай, которьй шире, чем случай полного подобия, хотя тоже является частным. Напомним, что на самом деле исследователь заинтересован не в пределе, а в асимптотике при больших, хотя и конечных, значениях $\Pi_{1}$. Итак, если нет конечного ненулевого предела величины $\Phi\left(\Pi_{1}\right)$ при $\Pi_{1} \rightarrow \infty$, но асимптотика $\Phi\left(\Pi_{1}\right)$ при $\Pi_{1} \rightarrow \infty$ имеет степенной характер, $\Phi=C \Pi_{1}^{\alpha}$, то мы получаем масштабньй закон как будто бы того же вида, что и (10):

$$
a=C a_{1}^{p-\alpha p_{1}} \cdots a_{k}^{r-\alpha r_{1}} b^{\alpha} .
$$

Различие между (10) и (11) состоит в том, что в (11) показатели не могут быть получены с помощью размерностного анализа, поскольку число $\alpha$ априори неизвестно, и величина $b$ продолжает присутствовать в (11), хотя и в виде мономной комбинации с другими аргументами. Мы будем назьвать эти случаи неполным подобием. (На самом деле здесь представлен очень частньй случай неполного подобия, которьй нам понадобится ниже; по поводу обшего обсуждения мы отсылаем читателя к [13].)

4. Сильньй аргумент в пользу допущения неполного подобия для потоков в трубах имеется в книге Шлихтинга [14]. Наряду с полньм уважением к универсальному логарифмическому закону, в этой книге представлен ряд графиков для “поддержки 
предположения" о зависяшем от числа Рейнольдса степенном законе для распределения скорости в трубах (см. рис. 3). Эти графики показывают, что данные экспериментов Никурадзе [15], которые обычно рассматривались (в том числе и самим Прандтлем) как подтверждение универсального логарифмического закона (6), могут быть представлены с поразительной точностью практически во всем поперечном сечении трубы степенным законом с показателем, зависящим от числа Рейнольдса.
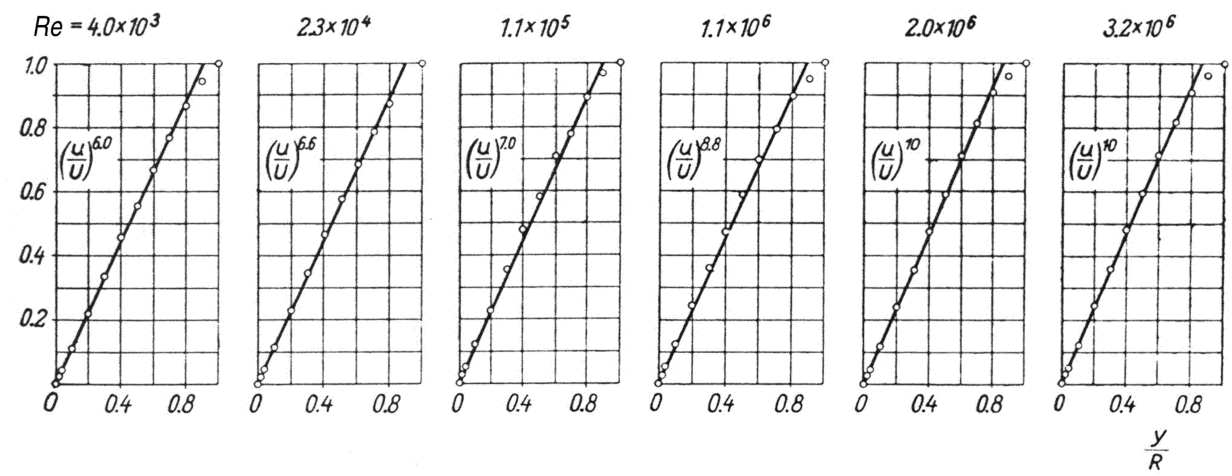

Рис. 3. Распределение скоростей в гладких трубах может быть представлено с хорошей точностью степенньм законом с зависящим от числа Рейнольдса показателем практически по всему поперечному сечению трубы. Здесь $R=d / 2$ и $U-$ скорость на центральной прямой (Шлихтинг [14])

Поэтому естественный шаг состоит в том, чтобы предположить, что в случае ограниченных стенками турбулентных потоков со сдвигом нет полного подобия, соответствуюшего гипотезе фон Кармана, и предложить следуюшую по сложности гипотезу:

ПЕРВАЯ ГИПОТЕЗА: имеется неполное подобие градиента средней скорости по параметру $у / \delta=u_{*} y / \nu=\eta$, и никакого подобия по числу Рейнольдса.

В соответствии с этой гипотезой, влияние вязкости сохраняется при произвольно больших числах Рейнольдса во всем объеме потока, а не только в вязком подслое. После работ Клайна, Рейнольдса и их групшы в Стэнфорде (см. [16] и много последующих работ этих авторов) физический механизм вторжения вязкости в ядро потока ясен: это - вторжение вихрей из вязкого подслоя в основное тело потока. Действительно замечательно, что Прандтль предчувствовал этот механизм гораздо раньше. В дискуссии, последовавшей немедленно за лекцией фон Кармана [1], Прандтль сказал, что несогласие с формулами фон Кармана может быть “связано с действием вязкости также и во внутренней части потока, т.е. вязкости, вызванной прожилками, из которых состоит пристеночный ламинарньй слой, и которьй в этом случае далеко входит во внутреннюю часть потока". Насколько известно автору настоящей статьи, этот комментарий никогда не повторялся ни самим Прандтлем, ни кем-нибудь другим из внутреннего круга Прандтля!

Неполное подобие означает, что вязкость входит в формулу только в комбинации с другими параметрами, управляющими турбулентностью. Согласно первой гипотезе, для очень большого Re функция $\Phi$, входящая в основное уравнение (4) при больших $\eta=y / \delta=u_{*} y / \nu$, должна быть степенной функцией своего аргумента $y / \delta=\eta$, причем 
не делается никаких специальных предположений о каком бы то ни было типе подобия по числу Рейнольдса Re, так что

$$
\Phi\left(\frac{y}{\delta}, \operatorname{Re}\right)=A(\operatorname{Re})\left(\frac{y}{\delta}\right)^{\alpha(\operatorname{Re})},
$$

где $A(\mathrm{Re})$ и $\alpha(\mathrm{Re})$ - некоторые безразмерные функции, подлежашие определению, a $\delta=\nu / u_{*}$.

Из (12) и (4) получаем

$$
\partial_{y} \phi=\frac{1}{y} A(\operatorname{Re})\left(\frac{u_{*} y}{\delta}\right)^{\alpha(\operatorname{Re})}
$$

где, напомним, $\phi=u / u_{*}$.

Наша вторая гипотеза состоит в приниипе исчезающей вязкости:

ВТОРАЯ ГИПОТЕЗА: градиент средней скорости стремится к хорошо определенному пределу, когда вязкость стремится к нулю.

Согласно этому принципу, можно разложить $A(\operatorname{Re})$ и $\alpha(\operatorname{Re})$ в ряд по малому параметру $\varepsilon(\mathrm{Re})$, стремяшемуся к нулю при $\mathrm{Re} \rightarrow \infty$, и сначала сохранить два слагаемых: $A=A_{0}+A_{1} \varepsilon, \alpha=\alpha_{0}+\alpha_{1} \varepsilon$, где $A_{0}, A_{1}, \alpha_{0}$ и $\alpha_{1}$ должны быть (по логике вывода) универсальными постоянными. Из (13) получаем

$$
\partial_{y} \phi=\frac{1}{y}\left(A_{0}+A_{1} \varepsilon\right)\left(\frac{y}{\delta}\right)^{\alpha_{0}+\alpha_{1} \varepsilon} .
$$

Когда вязкость (и, следовательно, масштаб длины $\delta$ ) стремится к нулю, корректно определенньй предел величины $\partial_{y} \phi$ может существовать только при $\alpha_{0}=0$. Следовательно, согласно второй гипотезе, значение $\alpha_{0}$ равно нулю. Кроме того, соотношение (14) можно представить в виде

$$
\partial_{y} \phi=\frac{1}{y}\left(A_{0}+A_{1} \varepsilon\right) \exp \left(\alpha_{1} \varepsilon \ln \frac{y}{\delta}\right) .
$$

Мальй параметр $\varepsilon$ является функцией от $R e$, обрашаюшейся в нуль при $R e=\infty$. Сейчас мы обсудим нечто очень важное. Соотношение (15) показьвает, что если $\varepsilon$ стремится к нулю при $\operatorname{Re} \rightarrow \infty$ быстрее, чем $1 / \ln R e$, то показатель экспоненты в (15) стремится к нулю, и мы возврашаемся к случаю полного подобия. Как мы видели, эксперименты показьвают, что это не так. Если $\varepsilon$ стремится к нулю медленнее, чем $1 / \ln \operatorname{Re}$, то не существует никакого хорошо определенного предела градиента скорости, когда вязкость стремится к нулю, и мы получаем противоречие с нашим вторьм предположением - принципом исчезающей вязкости. Таким образом, единственньй выбор, согласованньй с основньми предположениями, имеет вид $\varepsilon=1 / \ln R e$, и, интегрируя (15), мы получаем масштабный закон для распределения скорости в промежуточной области:

$$
\phi=\frac{u}{u_{*}}=\left(C_{0} \ln \operatorname{Re}+C_{1}\right)\left(\frac{y}{\delta}\right)^{\alpha_{1} / \ln \operatorname{Re}} .
$$

Заметим, что здесь было использовано дополнительное соотношение $\phi(0)=0$. Это условие является дополнительным предположением, подтвержденным экспериментами (см. рис. 3$)$, которое не следует из условия отсутствия скольжения $u(0)=0$, потому 
что граница $y=0$ находится вне области применимости асимптотического соотношения (15).

Постоянные $C_{0}, C_{1}$ и $\alpha_{1}$ были получены путем сравнения закона (16) с данными классических экспериментов Никурадзе [15], вьполненных под прямьм управлением Прандтля. Были получены следующие значения:

$$
C_{0}=\frac{1}{\sqrt{3}}, \quad C_{1}=\frac{5}{2}, \quad \alpha_{1}=\frac{3}{2} .
$$

Следовательно, окончательньй масштабный закон для распределения скоростей в основной промехуточной области трубы (см. рис. 1) принимает вид

$$
\phi=\left(\frac{\sqrt{3}+5 \alpha}{2 \alpha}\right) \eta^{\alpha}, \quad \alpha=\frac{3}{2 \ln \operatorname{Re}}, \quad \phi=\frac{u}{u_{*}}, \quad \eta=\frac{u_{*} y}{\nu} .
$$

Отметим отчетливое качественное отличие между случаями полного подобия (уравнение (6)) и неполного подобия (уравнение (18)). В первом случае на традиционной плоскости $\ln \eta, \phi$ экспериментальные точки должны сгущаться вдоль одной прямой линии универсального логарифмического закона. Во втором случае экспериментальные точки должны занимать область в плоскости $\ln \eta, \phi$, и каждому значению Re отвечает отдельная кривая. Экспериментальные данные, представленные на рис. 2 , подтверждают, что дела обстоят именно так. Поэтому масштабный закон (18) показывает, что вместо универсальной Re-независимой кривой для распределения скорости в плоскости $\ln \eta, \phi$, диктуемой универсальным логарифмическим законом, имеется семейство кривых в плоскости $\ln \eta, \phi$ c $\operatorname{Re}$ как параметром.

5. Ясный математический пример, предложенньй Чориным, показьвает, что происходит в поле скоростей ограниченных стенками турбулентных потоков со сдвигом. Рассмотрим семейство кривых

$$
\phi=\left(\ln \frac{d}{\delta}\right)\left(\frac{y}{\delta}\right)^{1 / \ln (d / \delta)}-2 \ln \frac{d}{\delta} .
$$

Здесь $\phi$-безразмерная функция, $d$ и $\delta$-постоянные параметры, имеюшие размерность длины, и $y \geqslant \delta$. Предположим, что $d$ фиксировано, а $\delta$ - параметр семейства.

Семейство (19) удовлетворяет обыкновенному дифференциальному уравнению

$$
\frac{d^{2} \phi}{d y^{2}}=\left(\frac{1}{\ln (d / \delta)}-1\right) \frac{1}{y} \frac{d \phi}{d y}
$$

и граничным условиям при $y=\delta$ :

$$
\phi(\delta)=-\ln (d / \delta),\left.\quad \frac{d \phi}{d y}\right|_{y=\delta}=\frac{1}{\delta} .
$$

Предположим теперь, что $d \gg \delta$, так что $1 / \ln (d / \delta)$ - мальй параметр. Кривые семейства (19) удовлетворяют следуюшему соотношению:

$$
y \partial_{y} \phi=\left(\frac{y}{\delta}\right)^{1 / \ln (d / \delta)}=\exp \left[\frac{\ln (y / d)+\ln (d / \delta)}{\ln (d / \delta)}\right],
$$


так что величина $y \partial_{y} \phi$ стремится к $е$ при $d / \delta \rightarrow \infty$ и при фиксированном $y / d$. Семейство (19) имеет огибаюшую

$$
\phi=\ln (y / d),
$$

для которой величина $y \partial_{y} \phi$ тоже постоянная, но другая, а именно, равная единице. Пренебрежем теперь в уравнении (20) малым параметром $1 / \ln (d / \delta)$ по сравнению с 1. Тогда это уравнение приводится к виду

$$
\frac{d^{2} \phi}{d y^{2}}=-\frac{1}{y} \frac{d \phi}{d y} .
$$

Решая это уравнение при граничных условиях (21), мы получаем одну не зависящую от $\delta$ “универсальную” кривую, огибающую (23), а не все семейство (19). Мы можем сказать, что, пренебрегая малым параметром в уравнении (20), мы "предотвратили" проникновение влияния параметра $\delta$ в основную область $y>\delta$, как делает и гипотеза фон Кармана, и это изменило всю картину.

Посмотрим на этот предмет с точки зрения размерностного анализа. Для производной $d \phi / d y$ размерностньй анализ дает

$$
\frac{d \phi}{d y}=\frac{1}{y} \Phi\left(\frac{y}{\delta}, \frac{d}{\delta}\right)
$$

где $\Phi$ - безразмерная функция. В нашем случае $\Phi=(y / \delta)^{1 / \ln (d / \delta)}$, так что при произвольно большом $y / \delta$ функция $\Phi$ не может быть заменена постоянной. Следовательно, влияние $\delta$ сохраняется при сколь угодно больших значениях $y / \delta$, и им нельзя пренебречь. Однако величина $\delta$ входит в итоговое уравнение (19) в специальной форме, в комбинации с $y$ и $d$, в связи с особой формой инвариантности задачи в целом.

6. Таким образом, повторяю, масштабный закон (18) показьвает, что вместо одной универсальный Re-независимой отдельной кривой для распределения скорости на плоскости $\ln \eta, \phi$ теперь в этой плоскости появляется семейство кривых, для которых число Рейнольдса является параметром. Однако семейство (18) имеет особое свойство автомодельности (самоподобия) и поэтому универсальности. Действительно, если вместо $\phi$ мы изобразим на оси координат величину

$$
\psi=\frac{1}{\alpha} \ln \frac{2 \alpha \phi}{\sqrt{3}+5 \alpha}, \quad \alpha=\frac{3}{2 \ln \operatorname{Re}},
$$

то из (18) следует, что $\psi=\ln \eta$, т.е. уравнение биссектрисы первого квадранта на плоскости $\ln \eta, \psi$. Это - ключевой момент: подтвердят ли это эксперименты, да или нет? Обрабатывая все доступные экспериментальные данные Никурадзе, я выразил в форме приведенных здесь таблиц (см. рис. 4) тот факт, что и на самом деле подавляюшее большинство экспериментальных точек для $\eta>30$ располагаются вдоль биссектрисы. Точки, соответствуюшие $\eta<30$, отклоняются от биссектрисы, и это естественно, поскольку масштабньй закон описывает распределение скоростей в промежуточной части поперечного сечения, а значения $\eta>30$ соответствуют вязкому подслою. Заметим, что имеется систематическое отклонение, а не разброс.

7. Масштабньй закон (18) позволяет решить вторую задачу, очерченную фон Карманом для потоков в трубах, - найти лобовое сопротивление. Лобовое сопротивление 


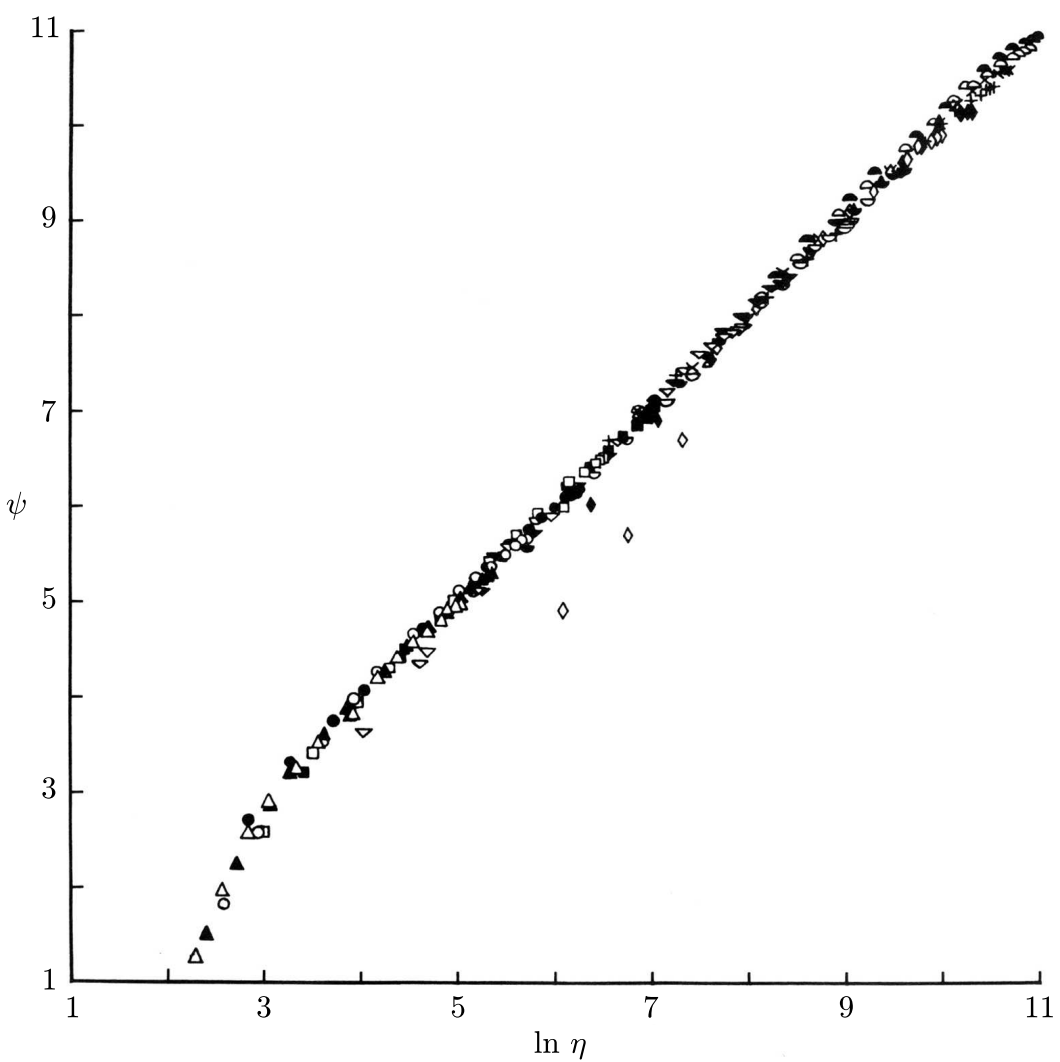

Рис. 4. Экспериментальные данные Никурадзе [15] в координатах $(\ln \eta, \psi)$ при $\eta>30$ лежат близко к биссектрисе первого квадранта, подтверждая масштабный закон.
(1) $\triangle, \quad \operatorname{Re}=4 \cdot 103$;
(3) $\quad \circ, \quad \operatorname{Re}=9.1 \cdot 103$;
(5) $\square, \quad \operatorname{Re}=2.33 \cdot 104$;
(7) $\nabla, \quad \operatorname{Re}=1.05 \cdot 105$;
(9) белый пнлуйкруг, $\operatorname{Re}=3.96 \cdot 105$;
(11) $\diamond, \quad \operatorname{Re}=1.11 \cdot 106$;
(13),$+ \quad \operatorname{Re}=1.959 \cdot 106$
(15) $\underset{\text { белый полуйкруг }}{\text { верий }}, \operatorname{Re}=2.79 \cdot 106$;

\begin{tabular}{|c|c|c|}
\hline $\begin{array}{l}(2) \\
(4) \\
(6) \\
(8)\end{array}$ & $\begin{array}{l}\boldsymbol{\Delta}, \\
\bullet \\
\dot{\boldsymbol{\theta}} \\
\vec{\nabla}\end{array}$ & $\begin{array}{l}\operatorname{Re}=6.1 \cdot 103 \\
\operatorname{Re}=1.67 \cdot 104 \\
\operatorname{Re}=4.34 \cdot 104 \\
\operatorname{Re}=2.05 \cdot 105\end{array}$ \\
\hline (10) & $\begin{array}{c}\text { нижний } \\
\text { черный по лукруг }\end{array}$ & $\operatorname{Re}=7.25 \cdot 105$ \\
\hline $\begin{array}{l}(12) \\
(14)\end{array}$ & & $\begin{array}{l}\operatorname{Re}=1.536 \cdot 106 \\
\operatorname{Re}=2.356 \cdot 106\end{array}$ \\
\hline & верхний & $\operatorname{Re}=3.24 \cdot 106$ \\
\hline
\end{tabular}

определяется напряжением на стенке $\tau$, и безразмерньй коэффициент лобового сопротивления определяется по правилу $\lambda=\tau /\left(\rho \bar{u}^{2} / 8\right)=8\left(u_{*}^{2} / \bar{u}^{2}\right)$, которое в литературе является сейчас общепринятым. Для определения среднего значения скорости $\bar{u}$ (по сечению) мы используем масштабный закон (18) и поэтому пренебрегаем отклонением распределения скорости от масштабного закона (18) и в вязком подслое, и вблизи оси. Мы получаем формулу для коэффичиента лобового сопротивления как функиии числа Рейнольдса:

$$
\lambda=\frac{8}{\Psi^{2 /(1+\alpha)}}, \quad \Psi=\frac{e^{3 / 2}(\sqrt{3}+5 \alpha)}{2^{\alpha} \alpha(1+\alpha)(2+\alpha)}, \quad \alpha=\frac{3}{2 \ln \operatorname{Re}} .
$$


Сравнение этого закона с независимом рядом экспериментов, где было определено трение, также показьвает поучительное согласие. Отклонения находятся в пределах нормального экспериментального рассеяния (см. [6], [7] и более ранние ссылки в этих работах).

Мы приходим к заключению, что масштабньй закон (18) с универсальньми, не зависяшими от числа Рейнольдса постоянными (17) и закон лобового сопротивления (27) точно описьвают турбулентные потоки в гладких трубах при больших числах Рейнольдса, и можно считать установленньм неполное подобие для таких потоков. Мы подчеркиваем, что значения постоянных (17) должны рассматриваться как фиксированные, нерегулируемые параметры.

8. Универсальный логарифмический закон окаменел до состояния догмы, стал одним из основных принщипов теории турбулентности и, в большой степени, опорой инженерной науки, поскольку он был подкреплен независимьм математическим выводом, основанным на, казалось бы, неопровержимых принципах. Этот вывод был предложен Изаксоном [17], Милликеном [18] и фон Мизесом [19].

Наш анализ показал, что вывод Изаксона-Милликена-фон Мизеса не вполне верен, и должен быть изменен с учетом влияния числа Рейнольдса (см. [6], [7]). В дополнение к расщеплению кривых, соответствуюших разньм числам Рейнольдса, характеристической особенностью масштабного закона (18) является наличие прямолинейных частей на этих кривых на плоскости $\ln \eta, \phi$. Именно это свойство позволяет модифицировать рассуждения Изаксона-Милликена-фон Мизеса. Это очевидно подкрепляется экспериментами Загарола (см. рис. 2).

Кроме того, семейство степенных законов (18), для которых Re является параметром, допускает огибаюшую (ср. пример Чорина, приведенньй в п. 5). Соотношение для огибающей получается в неявной форме исключением Re из уравнения (18) и уравнения, получаемого из (18) дифференцированием по Re. Огибаюшая имеет важное свойство: она практически неотличима в рабочей области $\ln \eta$ от прямой линии

$$
\phi=\frac{u}{u_{*}}=\frac{\sqrt{3} e}{2} \ln \eta+5.1 .
$$

Учитывая, что $2 /(\sqrt{3} e) \simeq 0.425$, мы видим, что прямая $(28)$ может быть отождествлена с традиционной формой универсального логарифмического закона. Поэтому, если изобразить экспериментальные точки, соответствуюшие различньм значениям $\operatorname{Re}$, на одном и том же графике на плоскости $\ln \eta, \phi$, что естественно для тех, кому посчастливилось верить в универсальньй логарифмический закон, то огибающая будет обнаружена. Наглядное впечатление от огибающей, когда экспериментальные данные изображены на плоскости $\ln \eta, \phi$, увеличивается оттого, что измерения при очень малых значениях $\eta$, где различия между степенными законами и огибающей могут также быть заметны, отсутствуют в связи с экспериментальными трудностями.

Подведем итог: подтверждение универсального логарифмического закона экспериментом для потоков в трубах вьглядит всего лишь иллюзией. Есть еше один факт в пользу этого заключения: асимптотический анализ показьвает, что при больших значениях $\operatorname{Re}$ наклоны прямолинейных участков кривых на плоскости $\ln \eta$, $\phi$ отклоняются на величину порядка $\sqrt{e}$ от наклона огибающей $(28)$, и этот факт тоже находится в согласии с экспериментом (см. рис. 2). 
9. По самой логике своего вывода, масштабный закон (18) должен выполняться для всех ограниченных стенками потоков со сдвигом, а не только для потоков в трубах.

Однако здесь возникает следующий вопрос: что должно быть определением числа Рейнольдса для таких потоков, которое позволит использовать эти числа в масштабном законе (18)? Этот вопрос был бессодержателен, пока исследователь верил в универсальный логарифмический закон: действительно, если закон не зависит от Re, то определение числа Re несушественно. Но положение совершенно меняется, если закон зависит от числа Re!

Ниже мы рассматриваем пограничные слои и покажем, что закон (18) описывает и эти потоки, если число Рейнольдса определено подходящим образом.

Пограничные слои с нулевым градиентом давления были исследованы многими экспериментаторами в течение последних десятилетий. Обычно в качестве числа Рейнольдса для таких потоков выбирают величину $\operatorname{Re}_{\theta}=U \theta / \nu$. Здесь $U-$ скорость свободного потока, а $\theta$ - толщина потери импульса, величина, вычисляемая с помошью интегрирования профиля скорости. Этот выбор довольно произволен, и определенно нельзя априори ожидать справедливости масштабного закона (18) для $\operatorname{Re}=\operatorname{Re}_{\theta}$. Hо в чем состоит правильньй выбор числа Рейнольдса для пограничных слоев?

Для ответа на этот вопрос мы должны прежде всего подтвердить, что в промежуточном слое турбулентного пограничного слоя, примыкающего к вязкому подслою, выполняется некоторый определенный масштабный закон. С этой целью (подробности приведены в [9]) все доступные экспериментальные данные, которые обычно представляются в традиционной плоскости $\ln \eta, \phi$, были перенесены на билогарифмическую плоскость $\lg \eta, \lg \phi$. Результат оказался поучительньм: без каких-либо исключений, для всех исследованных потоков были получены прямолинейные участки для области (I), примыкаюшей к вязкому подслою (см. те же примеры на рис. 5). Прямая линия (I) соответствует масштабному закону

$$
\phi=A \eta^{\alpha}
$$

где коэффициенты $A$ и $\alpha$ были получены в каждом случае с помощью статистической обработки данных.

Мы берем число Рейнольдса в виде $\operatorname{Re}=U \Lambda / \nu$, где, повторяем, $U-$ скорость свободного потока, а $\Lambda$ - некоторьй масштаб длины. Вопрос в том, существует ли единичньй масштаб длины $\Lambda$, который в промежуточной области (I) пограничного слоя играет ту же роль, что и диаметр трубы для потока в трубе, т.е. такой, что масштабньй закон (18) выполняется в промежуточной области (I)? Чтобы ответить на этот вопрос, были вычислены два значения $\ln \operatorname{Re}_{1}$ и $\ln \mathrm{Re}_{2}$ с помошью решения уравнений, подсказываемых масштабным законом (18):

$$
\frac{1}{\sqrt{3}} \ln \operatorname{Re}_{1}+\frac{5}{2}=A, \quad \frac{3}{2 \ln \operatorname{Re}_{2}}=\alpha,
$$

где, повторяем, $A$ и $\alpha$ были получены статистической обработкой экспериментальных данных в первой промежуточной автомодельной области. Вопрос вьглядел так: близки ли значения $\ln \operatorname{Re}_{1}$ и $\ln \operatorname{Re}_{2}$, получаемые при решении двух различных уравнений? Еще раз: да или нет?

Анализ всех доступных экспериментальных данных [9] показал, что значения $\ln \mathrm{Re}_{1}$ и $\ln \operatorname{Re}_{2}$ и в самом деле близки и, как правило, разность между ними не превосходит одного-двух процентов. Поэтому можно ввести среднее число Рейнольдса Re = 

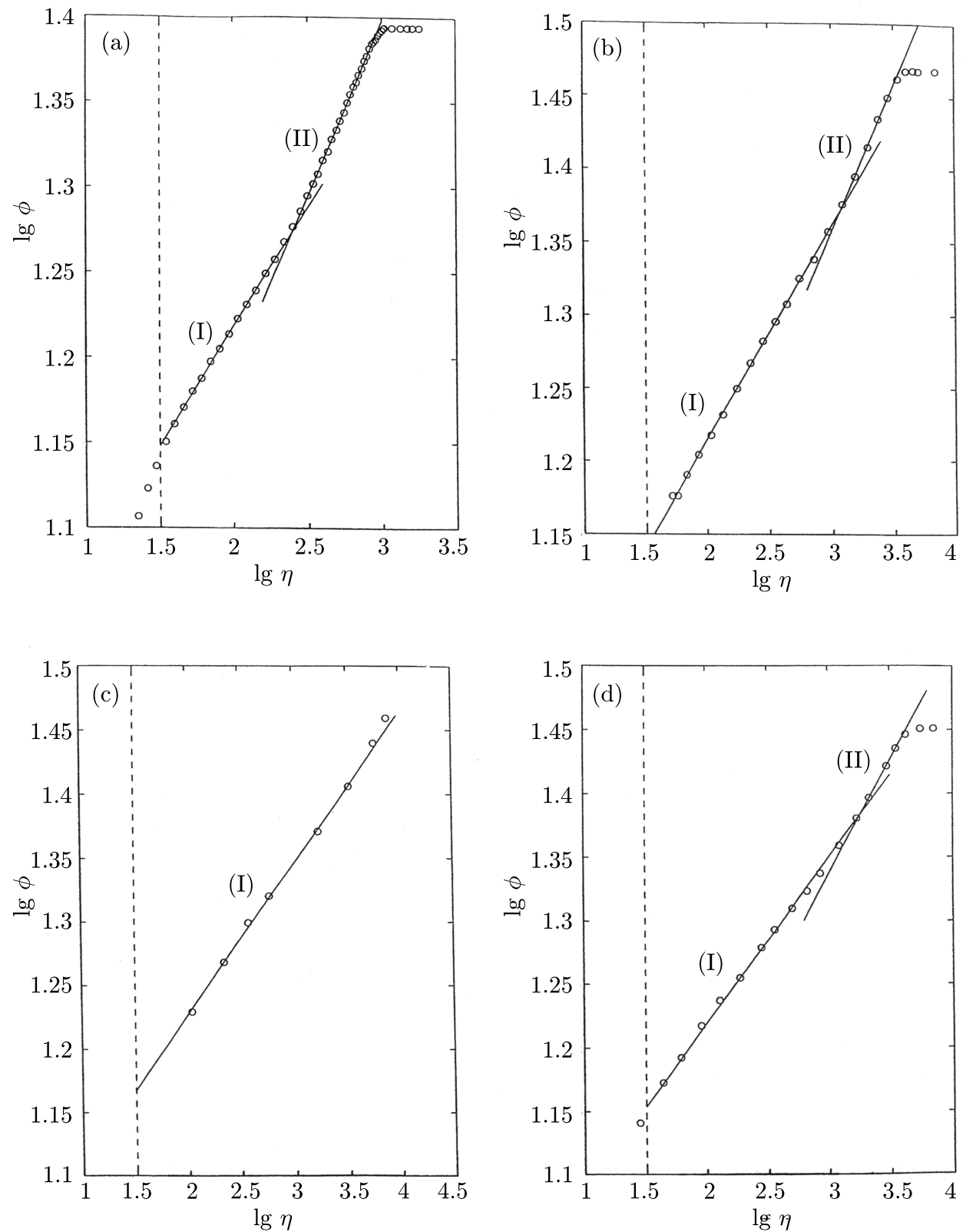

Рис. 5. (а) Эксперименты Эрма и Жубера [20], $\operatorname{Re}_{\theta}=2$ 788. Обе автомодельные промежуточные области (I) и (II) хорошо видны.

(b) Эксперименты Крогстада и Антониа [21], $\operatorname{Re}_{\theta}=12$ 570. Обе автомоделшные промежуточные области (I) и (II) хорошо видны.

(c) Эксперименты Петри, Фонтена, Соммера и Бругара, полученные сканированием графиков в работе Фернхольца и Финли [22], $\operatorname{Re}_{\theta}=35530$. Первая автомодельная область (I) проявилась; вторая автомодельная область не проявилась.

(d) Эксперименты Смита, полученные сканированием графииков в работе Фернхольца и Финли [22], $\operatorname{Re}_{\theta}=12990$. Первая автомодельная область (I) хорошо видна, вторая область (II) может быть проявлена 
$\sqrt{\operatorname{Re}_{1} \operatorname{Re}_{2}}, \ln \operatorname{Re}=\left(\ln \operatorname{Re}_{1}+\ln \operatorname{Re}_{2}\right) / 2$, как оценку для эфффективного числа Рейнольдса пограничного слоя потока. После этого мы должны проверить масштабньй закон (18) в его универсальной форме

$$
\psi=\frac{1}{\alpha} \ln \left(\frac{2 \alpha \phi}{\sqrt{3}+5 \alpha}\right)=\ln \eta
$$

используя экспериментальные данные для $\phi$ и $\eta$ и предполагая, что $\alpha=3 /(2 \ln \operatorname{Re})$ и $\ln R e=\left(\ln \operatorname{Re}_{1}+\ln \operatorname{Re}_{2}\right) / 2$. Согласно (31), если наше построение корректно, то в координатах $\ln \eta, \psi$ экспериментальные точки должны сбиваться к биссектрисе первого квадранта, как это было в случае потока в трубах. На рис. 6 представлены примеры такой обработки данных, которые показьвают, что именно так дело и обстоит. Результаты подобной обработки других данных можно найти в [9]. Мы заключаем, что масштабный закон (18) дает точное описание распределения средней скорости в автомодельной области (I), примыкающей к вязкому подслою, для большого семейства потоков в пограничных слоях с нулевьм градиентом давления.

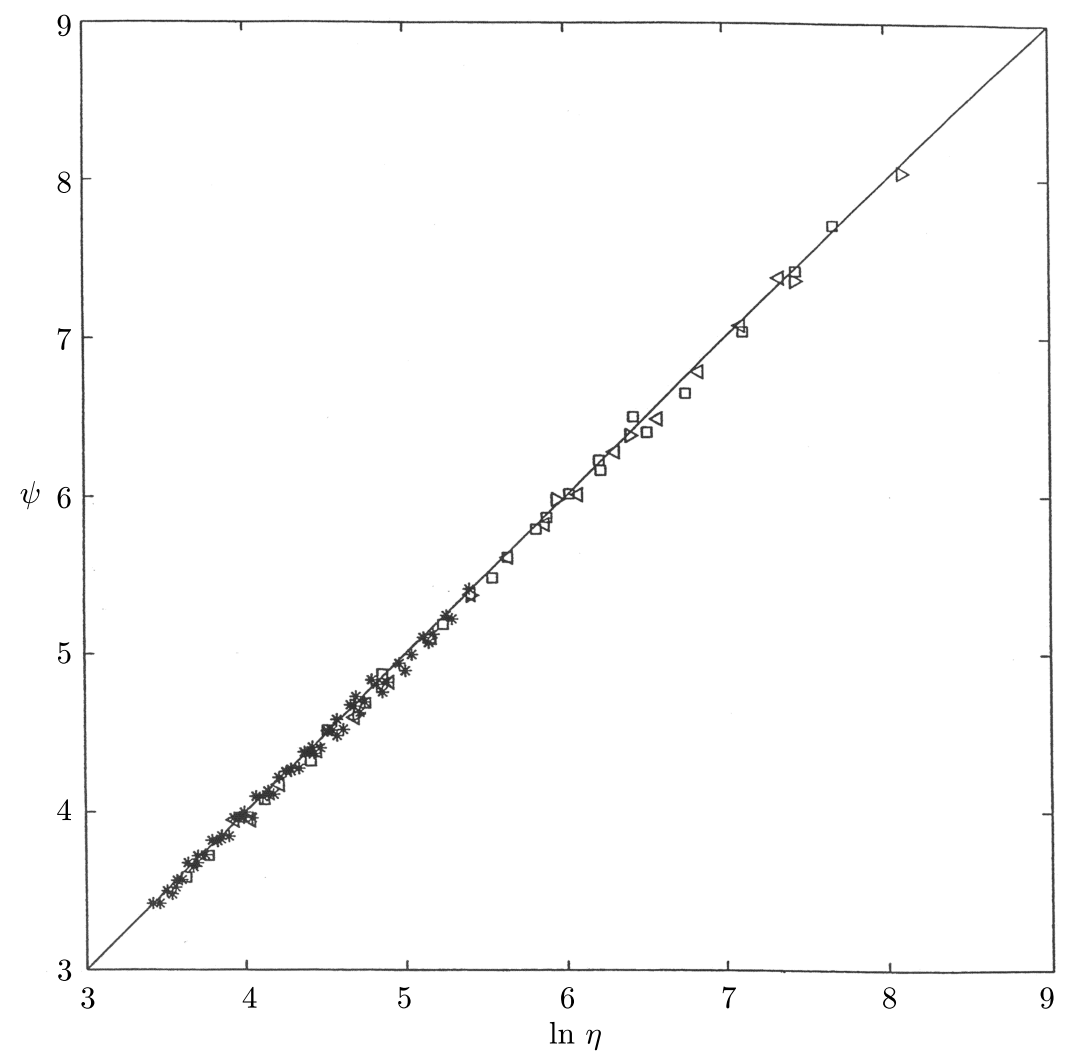

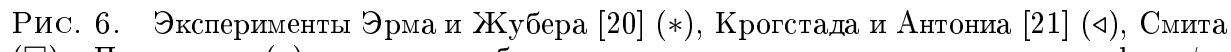
$(\square)$ и Петри и др. ( $\triangleright)$ сгущаются к биссектрисе первого квадранта на плоскости $\ln \eta, \psi$ в соответствии с универсальной формой (31) масштабного закона (25) 
Для потоков, в которых интенсивность турбулентности свободного потока невысока, была обнаружена вторая автомодельная область (II) (см. [9]). Распределение скорости в этой области, примыкающей к свободному потоку, тоже описывается масштабным законом

$$
\phi=B \eta^{\beta}
$$

с параметрами, отличаюшимися от параметров закона (29). Пересечение этих двух автомодельных областей ясно выражено. Число Рейнольдса для области (I) было определено равенством $\operatorname{Re}=U \Lambda / \nu$, где $U$ - скорость свободного потока, а $\Lambda$ - масштаб длины, определяемьй вьшеописанной процедурой. Анализ показал (см. [23]), что для исследуемых потоков величина $\Lambda$ приблизительно равна произведению множителя $1.5 \div 1.6$ на толшину пристеночной области, определяемой ясно очерченньм пересечением двух областей (I) и (II).

Справедливость масштабного закона (18) для нижних автомодельных областей потоков в пограничном слое является сильным аргументом в пользу справедливости этого закона в широком классе ограниченных стенками турбулентных потоков со сдвигом при больших числах Рейнольдса.

Природа второй автомодельной области, примыкающей к свободному потоку, еще не прояснена. Для потоков в пограничных слоях с нулевыми градиентами давления и с низкой турбулентностью свободного потока показатель степени $\beta$ в масштабном законе оказался близким к 0.2. Данные для потоков с ненулевыми градиентами давления сушественно менее многочисленны. Недавно данные Марушича и Перри [24] появились в Интернете в цифровой форме. Обработка этих данных (см. [25]) подтвердила структуру “ломаной линии” для распределения скорости в пограничном слое: две автомодельные области с отчетливым пересечением (см. рис. 7). Она также показала существенную вариативность показателя $\beta$. Оказалось, что в дополнение к параметру Re, управляющему структурой области (I), показатель $\beta$ и коэффициент $B$ в масштабном законе (32) зависят от безразмерного параметра

$$
P=\nu \partial_{x} p /\left(\rho u_{*}^{3}\right),
$$

где $\partial_{x} p-$ градиент давления.

В соответствии с основными предположениями моделей турбулентных пограничных слоев, которые были ранее предложены Клаузером [26] и Коулзом [27] и которые широко принимаются и используются, переход от потока в пристеночной области, описьваемого универсальньм логарифмическим законом, к внешнему потоку является гладким. В согласии же с новой моделью, представленной вьше, если турбулентность во внешнем потоке мала, то промежуточная область между вязким подслоем и внешним потоком состоит из двух зависящих от числа Рейнольдса автомодельных областей, отделенных ясно очерченной областью. Если внешний поток турбулизован, то переход от пристеночной области, где справедлив закон (18), к внешнему потоку является гладким, но поток вне пристеночной области испытьвает сильное влияние турбулентности внешнего потока, которое должно быть явным образом включено в анализ в этой области.

В заключение я могу сказать следующее. Полученные результаты значимы, поскольку инженеры давно знали, что выводы, полученные на основе универсального логарифмического закона, например, коэффициент лобового сопротивления, недостоверны, и вместо этого использовали “исправленные” эмпирические функции, которые 


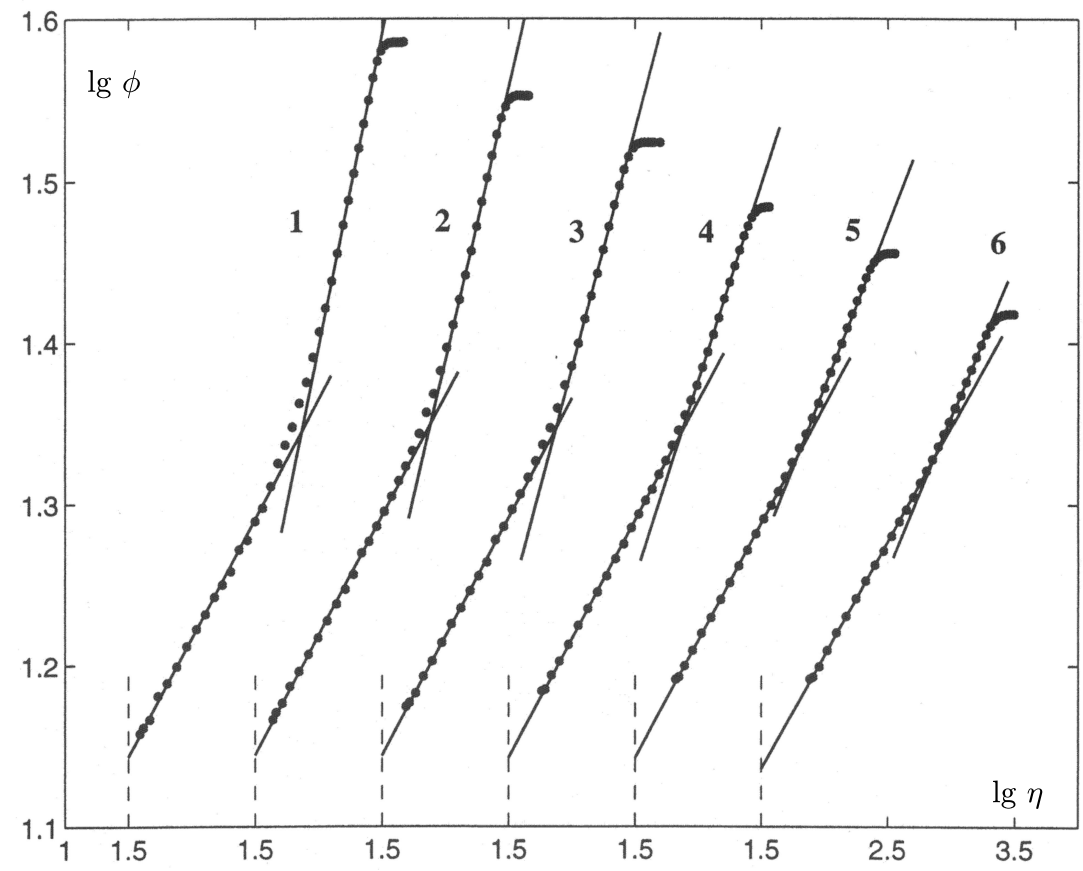

Рис. 7. Профили средних скоростей в билогарифмических координатах в ряде экспериментов Марушича [24] при $U=30 \mathrm{~m} / \mathrm{s}$, против градиента давления.

(1) $\operatorname{Re}_{\theta}=19133, \ln \operatorname{Re}_{\Lambda}=8.83, \quad P=7.04 \cdot 10^{-3}, \beta=0.388$

(2) $\operatorname{Re}_{\theta}=16584, \ln \operatorname{Re}_{\Lambda}=10.18, \quad P=5.79 \cdot 10^{-3}, \beta=0.346$

(3) $\operatorname{Re}_{\theta}=14208, \ln \operatorname{Re}_{\Lambda}=10.20, \quad P=4.2 \cdot 10^{-3}, \quad \beta=0.306$

(4) $\operatorname{Re}_{\theta}=10997, \ln \operatorname{Re}_{\Lambda}=10.31, \quad P=2.86 \cdot 10^{-3}, \quad \beta=0.247$

(5) $\operatorname{Re}_{\theta}=8588, \quad \ln \operatorname{Re}_{\Lambda}=10.323, P=1.75 \cdot 10^{-3}, \beta=0.207$

(6) $\operatorname{Re}_{\theta}=6430, \quad \ln \operatorname{Re}_{\Lambda}=10.51, \quad P=0, \quad \beta=0.190$

Хорошо видна структура “ломаной линии” в профилях и ясно различимы области I и II

соответствовали данным; теперь эмпирические соответствия могут быть выведены из лучшего закона. Однако основное значение этой работы состоит в ее влиянии на теорию: она связьвает и также утверждает наше понимание ключевых проблем турбулентности и имеет многочисленные следствия.

\section{СПИСОК ЛИТЕРАТУРЫ}

[1] Th. von Kármán. Mechanische Ähnlichkeit und Turbulenz // Proceedings of the Third International Congress on Applied Mechanics (Stockholm, 1930). V. 1 / ed. C. W. Oseen and W. Weibull. AB Sveriges Lifografska Tryckenier, 1931. P. 85-93.

[2] L. Prandtl. Zur turbulenten Strömung in Röhren und längs Platten // Ergeb. Aerodyn. Versuchsanst. Göttingen. 1932. V. 4. P. 18-29.

[3] А. Н. Колмогоров. Локалшная структура турбулентности в несжимаемой вязкой жидкости при очень больших числах Рейнольдса // Докл. АН СССР. 1941. Т. 30. № 4. C. 299-303. 
[4] А. М. Обухов. О распределении энергии в спектре турбулентного потока // Докл. АН CCCP. 1941. T. 32. № 1. С. 22-24.

[5] А. Н. Колмогоров. Избранные труды. Математика и механика. М.: Наука, 1985.

[6] G. I. Barenblatt, A. J. Chorin, V. M. Prostokishin. Scaling laws in fully developed turbulent pipe flow // Appl. Mech. Rev. 1997. V. 50. P. 413-429.

[7] A. J. Chorin. New perspectives in turbulence // Quart. Appl. Math. 1998. V. 56. № 4. P. $767-785$.

[8] G.I. Barenblatt. Scaling laws for turbulent wall-bounded shear flows at very large Reynolds numbers // J. Engrg. Math. 1999. V. 36. № 4. P. 361-384.

[9] G.I. Barenblatt, A.J. Chorin, V.M. Prostokishin. Self-similar intermediate structures in turbulent boundary layers at large Reynolds numbers // J. Fluid Mech. 2000. V. 410. P. 263-283.

[10] Л. Д. Ландау, Е. М. Лифшшиц. Теоретическая физика. Т. 6: Гидродинамика. М.: Наука, 1986

[11] А. С. Монин, А. М. Яглом. Статистическая гидромеханика. Т. 1: Механика турбулентности. М.: Наука, 1965.

[12] M. V. Zagarola. Mean flow scaling in turbulent pipe flow // $\mathrm{PhD}$ Thesis. Princeton: Princeton Univ. Press, 1996.

[13] G.I. Barenblatt. Scaling, Self-Similarity, and Intermediate Asymptotics. Cambridge: Cambridge Univ. Press, 1996.

[14] H. Schlichting. Boundary Layer Theory. New York: McGraw-Hill, 1968.

[15] J. Nikuradse. Gesetzmässigkeiten der turbulenten Strömung in glatten Röhren // VDI Forschungscheftg № . 356, 1932.

[16] S. J. Kline, W. C. Reynolds, F. A. Schraub, P. W. Rundstadtler. The structure of turbulent boundary layers // J. Fluid Mech. 1967. V. 30. № 4. P. 741-774.

[17] A. Izakson. Formula for the velocity distribution near a wall // J. Exp. Theor. Physics. 1937. V. 7. P. 919-924.

[18] C. B. Millikan. A critical discussion of turbulent flows in channels and circular tubes // Proceedings of the Fifth International Congress on Applied Mechanics (Cambridge, 1938) / ed. J.P. den Hartog and H. Peters. New York/London: Wiley/Chapman \& Hall, 1939. P. 386-392.

[19] R. von Mises. Some remarks on the laws of turbulent motion in tubes // Th. von Kármán, Anniversary Volume. Pasadena: CalTech Press, 1941. P. 317-327.

[20] L. P. Erm, P. N. Joubert. Low Reynolds-number turbulent boundary layers // J. Fluid Mech. 1991. V. 230. P. 1-44.

[21] P.- Å. Krogstad, R. A. Antonia. Surface roughness effects in turbulent boundary layers // Exp. in Fluids. 1999. V. 27. P. 450-460.

[22] H. H. Fernholz, P. J. Finley. The incompressible zero-pressure gradient turbulent boundary layer: an assessment of the data // Progr. Aeros. Sci. 1996. V. 32. P. 245-311.

[23] G.I. Barenblatt, A. J. Chorin, V.M. Prostokishin. Characteristic length scale of the intermediate structure in zero-pressure-gradient boundary layer flow // Proc. Natl. Acad. Sci. USA. 2000. V. 97. № 8. P. 3799-3802.

[24] I. Marušić, A. E. Perry. A wall-wake model for the turbulence structure of boundary layers. Part 2. Further experimental support // J. Fluid Mech. 1995. V. 298. P. 389-407; http://www.mame.mu.oz.au/ivan.

[25] G. I. Barenblatt, A. J. Chorin, V. M. Prostokishin. A model of a turbulent boundary layer with a nonzero pressure gradient // Proc. Natl. Acad. Sci. USA. 2002. V. 99. № 9. P. 5772-5776.

[26] F. H. Clauser. The turbulent boundary layer // Adv. Appl. Mech. 1956. V. 4. P. 2-52.

[27] D. E. Coles. The law of the wake in the turbulent boundary layer // J. Fluid Mech. 1956. V. 1. № 3. P. 191-226.

University of California at Berkeley, 